\title{
MOUD Provision in Correctional Settings During Time of COVID-19: Prevention and Solutions
}

\author{
Nickolas Zaller, PhD and Lauren Brinkley-Rubinstein, PhD
}

Correctional settings can be vectors of infectious diseases due to overcrowding, unsanitary living conditions, and very little capacity to engage in social distancing. In the US, COVID-19 outbreaks were first identified in the New York City and Cook County jails, with infection rates far exceeding community rates. Each day new cases are being identified across the country in correctional facilities. People who are incarcerated are at increased risk of experiencing severe COVID-19 symptoms because of the increased prevalence of other underlying illnesses. Jails and prisons have begun initiating facility-level policies to help stop the spread of COVID-19. As a result, correctional agencies have reoriented staff to stem transmission in their facilities. This could translate into limited resources for other programming such as medications for opioid use disorder (MOUD) programs. In this commentary, we highlight risk mitigation practices for delivering MOUD in correctional settings during COVID-19 and note how to ensure quality of care while still preparing for the possibility of future pandemics.

Key Words: criminal justice, MOUD, opioids

( $J$ Addict Med 2020;14: e290-e292)

T he United States (US) incarcerates more people than any other nation; nearly two-thirds of individuals in prisons may meet criteria for substance use disorder (SUD). ${ }^{1}$ Correctional settings can be vectors of infectious diseases due to overcrowding, unsanitary living conditions, and very little capacity to engage in social distancing. In the US, COVID-19 outbreaks were first identified in the New York City and Cook County jails, with infection rates far exceeding community rates. To date, correctional facilities account for 39 of the top 50 largest cluster outbreaks in the country. ${ }^{2}$ People who are

From the College of Public Health, University of Arkansas for Medical Sciences, Little Rock, AR (NZ); School of Medicine, University of North Carolina at Chapel Hill, Chapel Hill, NC (LBR).

Received for publication May 29, 2020; accepted September 17, 2020.

Drs. Zaller and Brinkley-Rubinstein were supported by the Foundation for Opioid Response Efforts (FORE). The authors, and not the Foundation, are solely responsible for the content of the manuscript.

The authors have no conflicts of interest to disclose.

Send correspondence to Nickolas Zaller, $\mathrm{PhD}$, Professor, Health Behavior and Health Education, University of Arkansas for Medical Sciences, Fay W. Boozman College of Public Health, 4301 West Markham \#820, Little Rock, AR 72205. E-mail: NDZaller@uams.edu.

Copyright (C) 2020 American Society of Addiction Medicine

ISSN: $1932-0620 / 20 / 1406-\mathrm{e} 290$

DOI: $10.1097 / A D M .0000000000000758$ incarcerated may be at increased risk of experiencing severe COVID-19 symptoms because of the increased prevalence of other underlying illnesses, including addiction. In response, jails and prisons have begun initiating facility-level policies to help stop the spread of COVID-19. ${ }^{3}$ As a result, correctional agencies have reoriented staff to stem transmission in their facilities. This could translate into limited resources for other programming such as medications for opioid use disorder (MOUD) programs. This is especially important given that an estimated $15 \%$ of all individuals incarcerated in US prisons and jails have an opioid use disorder (OUD). ${ }^{4}$ In this commentary, we highlight risk mitigation practices for correctional facilities delivering MOUD during COVID-19 and note how to ensure quality of care while still preparing for the possibility of future pandemics.

\section{RISK MITIGATION PRACTICES FOR DELIVERING MOUD IN CORRECTIONAL SETTINGS DURING THE COVID-19 PANDEMIC}

Facilities should strive to continue MOUD programming but may need to modify practices to accommodate staff shortages due to COVID-19. We acknowledge that some recommendations may be difficult to quickly and fully implement, but recommend the following be considered:

- Continue to screen for OUD at intake but consider shortening the OUD screener. Many facilities use a validated screening tool but administering those may create undue burden during this time. An alternative approach, though not necessarily clinically valid based on current known evidence, could include asking people 2 questions: (1) do you regularly take any opioids? and (2) are you currently have a prescription for any MOUD?

- Deliver medications cell-side when possible and practice social distancing in the medication line (and ensure medical staff dispensing MOUD have appropriate personal protective equipment). For facilities that are too large for cell-side dispensing, consider use of staggering dispensation so that not all individuals have to receive their medication at the same time.

- Engage in tele-medicine as needed. If tele-medicine equipment is not available, look into establishing relationships with community treatment providers who can provide telemedicine services in concert with MOUD and/or other healthcare.

- Continue to link to MOUD services in the community. Make the first appointment for MOUD as soon as possible 
postrelease and make sure to provide access to naloxone and other harm reduction resources upon release.

- Take note of new federal policy guidance relevant to MOUD provision in the community to inform discharge planning efforts.

- Engage in virtual peer support and virtual SUD/OUD educational programming related to MOUD and/or recovery support.

- Leverage increased housing support that may be available in your state due to COVID-19. For example, in North Carolina some COVID-19 response monies have been used to pay for hotel rooms for people being released from correctional facilities that may otherwise have unstable housing.

Although dispensing MOUD and in general, incarcerated people and staff should have access to personal protective equipment according to the Center for Disease Control and Prevention's guidelines.

\section{RISK MITIGATION PRACTICE IN FOCUS: TELEMEDICINE}

In the current pandemic, telemedicine should be used to the extent possible. Recent federal guidance allows for the expanded use of telemedicine for prescribing controlled substances, including buprenorphine. As of the date a federal emergency was declared (January 21, 2020), SAMHSA has lifted the requirement mandating an in-person physical evaluation for any patient who will be treated with buprenorphine. ${ }^{5}$ Therefore, individuals within an incarcerated setting may be allowed to access buprenorphine treatment remotely via telemedicine and/or can continue treatment via telemedicine post release. Importantly, to facilitate greater use of telemedicine, the Department of Health and Human Services has temporarily waived sanctions ${ }^{6}$ and HIPPA Privacy Rule penalties associated with using telemedicine applications such as FaceTime or Skype.

Although the new guidance around physical evaluations does not apply to patients being treated with methadone, current methadone patients on stable doses can be maintained via telemedicine as per the new SAMHSA guidelines. Additionally, in March of 2020 SAMHSA issued updated recommendations around take-home medications stipulating blanket exceptions for all stable patients to receive 28 days of take-home medications for OUD. ${ }^{7}$ And although the recommendations are intended for opioid treatment programs (OTPs), many are still applicable to correctional facilities which are providing MOUD (or which contract with community-based OTP providers). Furthermore, even if correctional facilities are not certified as OTPs but are providing buprenorphine to treat OUD, they should provide adequate medication (at least a 14-day supply) to individuals upon release. This is especially important given the known risk for overdose during the immediate period postrelease. And provider capacity to accept new patients in some communities may be limited during the current pandemic.

In addition to provision of medication management via telemedicine, psychosocial counseling and peer support can also be delivered remotely. Although ASAM guidance recommends waiving requirements for counseling associated with MOUD, ${ }^{8}$ many patients receive significant benefit from ongoing counseling support. As such, online support groups are being encouraged in the face of continued requirements of social distancing. Virtual support groups may be appropriate in some correctional settings in lieu of traditional SUD treatment groups provided sufficient space is available to allow for adequate social distancing (this may mean restricting group sizes). Virtual peer support can also be an important component of reentry as individuals seek to connect (or re-connect) to treatment and recovery support services during the post-release period.

\section{THE IMPORTANCE OF REENTRY FOR INDIVIDUALS ON MOUD}

One of the key challenges for incarcerated individuals with MOUD is reentry and connecting to services upon release from criminal justice settings. As noted by the current NIDA Director, Nora Volkow, many COVID-19 related risks among individuals with SUD are indirect ${ }^{9}$ and include factors such as housing instability, reduced access to healthcare (substance use treatment, recovery support psychiatric care, and other medical care to treat chronic physical comorbidities), and lack of social support. Importantly, most individuals released from correctional settings have one or more of these indirect risks which clearly emphasizes the critical importance of robust discharge planning, especially for individuals with SUD/OUD. These risks are further compounded by the ongoing stigma associated with both SUD and criminal justice involvement. Additionally, reentry planning needs to incorporate assistance in obtaining health insurance (specifically Medicaid for states that have expanded Medicaid eligibility) for individuals being released. Some states suspend rather than terminate Medicaid benefits upon incarceration which can greatly expedite continuation of Medicaid coverage postrelease. ${ }^{10}$ Additionally, legislation introduced in the US House of Representatives would allow for the reinstatement of Medicaid benefits up to 30 days before release from incarceration. ${ }^{11}$ As healthcare systems and workers continue to struggle to provide care to the waves of COVID19 patients, the potential necessity of rationing care has already been debated. It is imperative that decisions regarding care not be influenced by explicit or implicit biases against individuals who have a history of SUD and/or criminal justice involvement. Finally, reentry services may need to be expedited within the context of early release for some individuals. Therefore, correctional systems need to have in place protocols for rapid transition of individuals back into the community.

\section{CONTINUED RISK MITIGATION FOR THE CURRENT PANDEMIC AND ENSURING READINESS FOR THE NEXT}

The current COVID-19 pandemic has clearly demonstrated the need to invest in public health preparedness across all sectors of society. And although criminal justice settings are often under-resourced, there are important steps correctional facilities can take to prepare for the potential of future pandemics. Above, we highlight telemedicine as one of the best risk mitigation practices for continued delivery of MOUD during a pandemic such as COVID-19. The most important recommendation for both current risk mitigation and future pandemic preparedness is to develop and/or enhance 
correctional and community partnerships. Such partnerships can ensure that MOUD best-practices will be followed, to the extent possible, even in times of crisis. Importantly, many of the practices outlined in this policy brief can be implemented as part of standard or routine service provision within criminal justice settings. At a minimum, these should include the following:

- Expand the use of telemedicine for healthcare services, especially behavioral health to minimize potential disruptions to treatment services for individuals receiving MOUD;

- Develop protocols for alternative forms of administration of MOUD if populations need to be cohorted and/or quarantined;

- Develop specific reentry protocols to facilitate MOUD linkages that can be rapidly initiated/implemented during times of crisis. These protocols also need to consider social support services, especially housing, for those being released.

\section{STRENGTHENING PUBLIC HEALTH PARTNERSHIPS}

In addition to these points of guidance, it is imperative that correctional administrators continue to partner with public health practitioners to further educate policy-makers and the general public about the importance of focusing public health interventions in correctional settings, which has become especially clear in the context of the pandemic. Policymakers throughout the US need to thoughtfully implement policies, such as expanded correctional diversion programs for individuals with OUD, which will reduce the numbers of incarcerated individuals to avoid the alarming states of prison and jail outbreaks we have seen in correctional facilities during the current COVID-19 pandemic. Individuals with SUD in general and OUD in specific are disproportionately represented in correctional facilities and will therefore benefit the most from such policies.

\section{REFERENCES}

1. Center on Addiction, Behind Bars II: Substance Abuse and America's Prison Population, February 2010. Available at: https://www.centeronaddiction.org/addiction-research/reports/behind-bars-ii-substance-abuseand-america's-prison-population. Accessed September 15, 2020.

2. The COVID Prison Project. The COVID Prison Project website. Available at: www.covidprisonproject.com. Accessed August 7, 2020.

3. Wagner P, Widra E. No need to wait for pandemics: the public health case for criminal justice reform. Prison Policy Initiative.

4. National Academies of Sciences, Engineering, and Medicine. Medications for Opioid Use Disorder Save Lives. Washington, DC: The National Academies Press; 2019. Available at: https://doi.org/10.17226/25310. Accessed September 15, 2020.

5. Substance Abuse and Mental Health Services Administration. FAQs: Provision of Methadone and Buprenorphine for the Treatment of Opioid Use Disorder in the COVID-19 Emergency. See more here: https:// www.samhsa.gov/sites/default/files/faqs-for-oud-prescribing-and-dispensing.pdf. Accessed September 15, 2020.

6. Health and Human Services. Notification of Enforcement Discretion of Telehealth Remote Communications during the COVID-19 Nationwide Public Health Emergency. See more here: https://www.hhs.gov/hipaa/forprofessionals/special-topics/emergency-preparedness/notificationenforcement-discretion-telehealth/index.html. Accessed September 15, 2020.

7. Substance Abuse and Mental Health Services Administration. Opioid Treatment Program (OTP) Guidance. See more here: https://www.samhsa.gov/sites/default/files/otp-guidance-20200316.pdf. Accessed September 15, 2020.

8. American Society of Addiction Medicine. Caring for Patients during the COVID-19 Pandemic. See more here: https://www.asam.org/docs/ default-source/covid-19/telehealth-guidance.pdf?sfvrsn=9c6e53c2_2. Accessed September 15, 2020.

9. Volkow, ND. Collision of the COVID-19 and Addiction Epidemics, 2020. Annals of Internal Medicine. See more here: https://annals.org/aim/fullarticle/2764313/collision-covid-19-addiction-epidemics. Accessed September 15, 2020.

10. Kaiser Family Foundation State Health Facts. Available at: https:// www.kff.org/medicaid/state-indicator/states-reporting-corrections-relatedmedicaid-enrollment-policies-in-place-for-prisons-or-jails/?currentTimeframe $=0 \&$ sortModel=\%7B $\% 22$ colId $\% 22: \% 22$ Location $\% 22, \% 22$ sort $\% 22: \%$ 22asc\%22\%7D. Accessed September 15, 2020.

11. H.R. 1329- Medicaid Reentry Act. Available at: https://www.congress. gov/bill/116th-congress/house-bill/1329/text. Accessed September 15, 2020. 